\title{
Competencias Tecnológicas de Información y Comunicación en los docentes en formación de Física a nivel universitario
}

\author{
Information and Communication Technological Competences in Physics training \\ teachers at university level
}

\author{
Ricardo Javier Zamora Rengifo \\ ricardozamora87@gmail.com \\ Código ORCID: 0000-0002-0911-4413 \\ Unidad Educativa Liceo Doctor Víctor Manuel Ovalles, \\ Venezuela
}

La presente investigación tuvo como propósito describir las Competencias Tecnológicas de Información y Comunicación (TIC) de los docentes en formación de la mención de Física de la FACE -UC caso: estudiantes del Seminario Proyecto de Investigación y Trabajo Especial de Grado de la mención de Física en la facultad de Ciencias de la Educación de la Universidad de Carabobo. El estudio se enmarcó en una investigación del tipo descriptivo, bajo un diseño de campo no experimental y transeccional. La población estuvo constituida por diez (10) sujetos. Para la recolección de datos se utilizó un instrumento validado por juicio de expertos elaborado por Guarapano y Martínez (2012), se realizó el cálculo de la confiabilidad mediante el Alfa de Cronbach, el cual arrojó un coeficiente de 0,87 que indica ser altamente confiable, según Ruiz (2002). Del análisis de los resultados se evidenció que los docentes en formación afirman tener Competencias Tecnológicas positivas.

Palabras clave:

Competencias Tecnológicas; docente en formación; Física that the teachers in training affirm that they have positive Technological Competences.

The purpose of this research was to describe the Technological Competences of Information and Communication (ICT) of the teachers in training of the mention of Physics of the FACE -UC case: Seminar students Research Project and Special Degree Work of the mention of Physics at the Faculty of Education Sciences of the University of Carabobo. The study was framed in an investigation of the descriptive type, under a non-experimental and transectional field design. The population consisted of ten (10) subjects. For data collection, an instrument validated by expert judgment prepared by Guarapano and Martínez (2012) was used. The calculation of reliability was performed using Cronbach's Alpha, which yielded a coefficient of 0.87 , which indicates that it is highly reliable, according to Ruiz (2002). From the analysis of the results, it was evident 


\section{INTRODUCCIÓN}

El uso de las tecnologías de la información y la comunicación (TIC) en la escuela tienen ya una amplia trayectoria. Sin embargo, su incorporación sistemática como herramienta de enseñanza y aprendizaje ha sido mucho más reciente y lenta porque existe una distancia considerable entre los avances tecnológicos en la producción de software libre educativo y la aceptación, comprensión y uso de estos recursos por parte del docente en el aula.

Es posible decir que en el contexto actual la tecnología ocupa un lugar muy importante tanto en la escuela como en las empresas y el día a día. Esta es una era digital donde los niños manejan cómodamente las tecnologías desde temprana edad. En el ámbito educativo existe la creencia de que las escuelas que poseen la mayor calidad son aquellas que están mejor dotadas de tecnologías.

Sin embargo, no debe considerase solamente las tecnologías de las que dispone la institución, sino las competencias que poseen los docentes para valerse de ellas, recordando que muchos fueron instruidos bajo otros paradigmas y estrategias didácticas que posiblemente ya se encuentren en desuso. Al respecto, Galvis (1992) expresa lo siguiente:

Los profesores "dictadores de clases" quizás usan una metodología de enseñanza tradicional porque así fue como ellos aprendieron, o bien porque no han tenido oportunidad de entrar en contacto o de llevar a la práctica otros enfoques en que se conjuguen variedad de medios y actividades y en las que el docente asuma el rol de facilitador, antes de transmisor. En que se sientan cómodos con ellas, es posible que se decidan a intentar un cambio, en procura de superar las limitaciones que tiene en su actual forma de trabajar".

Actualmente, la sociedad en general se encuentra significativamente influenciada por la tecnología de la información y comunicación (TIC), razón por la cual se han generado cambios en la forma de vivir, actuar y pensar. Es un fenómeno que ha afectado la manera de comunicarse, de gestionar, trabajar, comprar, etc. Incluso ha cambiado la metodología del sistema educativo, en especial a nivel universitario.

En relación a Venezuela, los estudios realizados por la Universidad Nacional Abierta (caracterizada por una educación a distancia) orientados a diagnosticar los conocimientos y habilidades que poseen los docentes en el uso de las TIC, determinaron que en un alto porcentaje poseen solo algunas competencias básicas para el manejo de herramientas. En general, los docentes señalan que utilizan muy poco las herramientas telemáticas para comunicarse con los estudiantes, de manera que la asesoría presencial cara a cara predomina. En relación con la ofimática tienen conocimientos en el manejo de procesadores de texto, elaboración de presentaciones $y$, en menor proporción, hojas de cálculo. Los profesores señalan además, que desconocen las estrategias para potenciar la interacción (Corredor y Rincón, 2014, p. 104). Al respecto Cesar Coll (2001) señala: 
La incorporación de las TIC a la educación no transforman ni mejoran automáticamente los procesos educativos, pero en cambio sí que modifican sustancialmente el contexto en el que tienen lugar estos procesos y las relaciones entre sus actores y entre ellos y las tareas y contenidos de aprendizajes, abriendo así el camino a una eventual transformación en profundidad de dichos procesos que se producirá o no, y que supondrá o no una mejora efectiva, en función de los usos concretos que se haga de la tecnología. (p. 13)

En este sentido las Tecnologías de la Información y Comunicación (TIC) juegan un papel preponderante, entendiéndose estas como "la que giran en torno a tres medios básicos: la informática, la microelectrónica y las telecomunicaciones; pero giran, no solo de forma aislada, sino lo que es más significativo, de manera interactiva e interconectadas, lo que permite nuevas realidades comunicativas" (Cabero y Llorente, 2005)

No obstante, numerosos estudios de orden internacional, evidencian el estado de atraso de los educadores en cuanto al empleo de la TIC como mediadoras de la actividad docente, se observa que el énfasis de ellas es mas de orden instrumental y técnico. Sin embargo, se puede decir que algunos docentes muestran una actitud positiva hacia la adopción y uso de la tecnología por parte de los estudiantes, pero al mismo tiempo no perciben las ventajas de incorporarlas a su práctica docente. Por lo tanto, se debe insistir en la revisión de la capacitación del docente para un uso pedagógico de las TIC que pueda responder a este nuevo paradigma, en concordancia con Cabero y Llorente (2005):

Uno de los errores más
significativos que se ha cometido
en la formación del docente, es
que se ha tenido una visión
demasiado técnica e instrumental
en su formación. Por ponerlo en
términos muy concretos, se le ha
formado demasiado en que
conozca la utilización de Word, la
base de datos Acces, la hoja de
cálculo Excel, las presentaciones
colectivas en Power Point, o las
posibilidades del Linux, que está
ahora de moda, y poco en que se
sepan incorporarlos a la práctica
didáctica-curricular, y transformar
y crear entornos diferenciados
para el aprendizaje, y no
exclusivamente para tareas
administrativas. (p. 4)

Respondiendo a esta necesidad en la Facultad de ciencias de la educación de la Universidad de Carabobo, en el pensum se incluye en el octavo semestre la asignatura de Módulo de diseño de materiales educativos cuya finalidad es integrar criterios técnicos y pedagógicos en la producción de materiales didácticos en el área de formación profesional con apoyo de las nuevas tecnologías.

Sin embargo, en semestres posteriores al aplicar las competencias adquiridas en uso didáctico de las TIC, se evidencia poco dominio de ellas, por lo que se dificulta su uso en las asignaturas de investigación como: Seminario Proyecto de Investigación y Trabajo Especial de Grado. De allí surge la 
siguiente pregunta de investigación ¿Cuáles son las competencias tecnológicas de información y comunicación (TIC) que poseen los docentes en formación de las asignaturas: Seminario Proyecto de investigación y Trabajo Especial de grado de la Mención de Física de la Facultad de Ciencias de la Educación de la Universidad de Carabobo? Como respuesta a esta interrogante surge el objetivo general a continuación: Describir las competencias de información y comunicación (TIC) de los docentes en formación de las asignaturas: Seminario Proyecto de Investigación y Trabajo Especial de Grado de la Mención de Física en la Facultad de Ciencias de la Educación de la Universidad de Carabobo.

Para alcanzar dicho objetivo general se desglosaron los siguientes objetivos específicos: 1. Determinar las competencias tecnológicas de información y comunicación (TIC) de los docentes en formación de la asignatura Seminario Proyecto de Investigación. 2. Establecer las competencias tecnológicas de información y comunicación (TIC) de los docentes en formación de la asignatura Trabajo Especial de Grado.

De este modo la investigación se sitúa dentro de un conjunto de basamentos teóricos que la sustenta. A continuación, se presentan algunos trabajos relacionados con el desarrollo de competencias tecnológicas educativas y antecedentes de la investigación que aportaron información relevante para el desarrollo de este trabajo.

Para este caso se tomará a Arias (2012) quien define los antecedentes de investigación como los estudios previos a la investigación que se realizará, la cual deberá estar vinculada con el proyecto a realizar, donde se debe enfatizar o señalar el objetivo, aportes y hallazgos principales. A continuación, se presentan algunas investigaciones vinculadas con este trabajo y que han representado aportes valiosos a investigación:

En primer lugar, se tiene a Guarapano y Martínez (2012) y la investigación titulada "Competencias del Docente en formación en el uso de las tecnologías de información y comunicación caso: Estudiantes de Práctica Profesional III de la mención de Matemática en la Facultad de Ciencias de la Educación de la Universidad de Carabobo" la cual tuvo como propósito fundamental describir las competencias en cuanto al uso de la tecnología de la información y comunicación de los estudiantes de educación mención matemática de la Universidad de Carabobo.

Dicha investigación se fundamentó en la teoría de J. Sarramona (2004). La metodología se enmarcó en el tipo descriptivo de campo, no experimental, la población de estudio estuvo constituida por la totalidad de 45 estudiantes del 10mo semestre de los turnos mañana, tarde y noche de la universidad de Carabobo, cursantes de la práctica profesional III a quienes se les aplicó un cuestionario tipo Likert de 35 ítems con cuatro alternativas de respuesta. Se determinó que el 30\% de los docentes en formación implementan los sistemas informáticos de los programas básicos y actitudes necesarias con las TIC, los cuales permiten recrear ambientes organizadores de aprendizajes complejos, estimular el trabajo colaborativo, examinar 
materiales en diversos formatos de presentación y estimular la reflexión y la negociación.

En resumen, la investigación realizada enfatiza el conocimiento que poseen los docentes en formación, referente a las nuevas tecnologías y al uso que les dan a las mismas, obteniéndose como resultado una situación deficiente en el uso de las mismas. Por lo que esta investigación se tomó como punto de partida en la presente para estudiar las competencias en los docentes en formación de la mención de física en cuanto al uso de las TIC y se dispuso su instrumento por encontrarse este validado y presentar una alta confiablidad.

En este mismo orden de ideas Ordoñez, Góngora, Ordoñez, Rico, y Cerón (2010) de la universidad de Cali en Colombia en su trabajo especial de grado titulado "Implementación de la tecnología computacional en el aprendizaje de la matemática y la física", desarrollaron como objetivo principal el uso las TIC para el aprendizaje de la matemática y la física para alcanzar las competencias en las nuevas tecnologías de educación. Llegaron a la conclusión que las TIC facilitan el aprendizaje de los conceptos, la resolución de problemas y contribuye a desarrollar habilidades cognitivas.

El ultimo antecedente a mencionar está en Rosario, Lobo, Rivero, Briceño, y Villarreal (2011) de la Universidad de los Andes, Trujillo, Venezuela y su investigación: Las TIC para el proceso enseñanza aprendizaje en los laboratorios de Física en el nivel universitario en el estado Trujillo, Venezuela. En este estudio se observó que a nivel universitario existe el interés de incorporar las TIC en los procesos de enseñanza aprendizaje, sin embargo, aunque el uso de las mismas por parte de los docentes de los laboratorios de física es aparentemente muy extendido, su aplicación se limita en buena parte a las presentaciones multimedia $\mathrm{O}$ distribución de documentos.

Desde esta perspectiva, la UNESCO (2008) afirma que para vivir, aprender y trabajar con éxito en una sociedad cada vez más compleja, rica en información y basada en el conocimiento, los estudiantes y los docentes deben utilizar la tecnología digital con eficacia. En un contexto educativo sólido, las Tecnologías de la Información y comunicación (TIC) pueden ayudar a los estudiantes a adquirir las capacidades necesarias para llegar a ser:

- Competentes para utilizar tecnologías de la información.

- Buscadores, analizadores y evaluadores de información.

- Usuarios creativos y eficaces de herramientas de productividad.

- Comunicadores, colaboradores, publicadores y productores; y

- Ciudadanos informados, responsables y capaces de contribuir a la sociedad.

Por lo tanto, gracias a la utilización continua y eficaz de las TIC en procesos educativos, los estudiantes tienen la oportunidad de adquirir capacidades importantes en el uso de estas. El docente es la persona que desempeña el papel más importante en la tarea de ayudar a los estudiantes a adquirir esas capacidades. Además, es el responsable de diseñar tanto oportunidades de aprendizaje como el 
entorno propio en el aula que facilite el uso de las TIC por parte del estudiantado para aprender y comunicar. Por eso es fundamental que todos los docentes estén preparados para ofrecer información actualizada y conocimientos útiles a sus estudiantes.

Por consiguiente, tanto los programas de desarrollo profesional para docentes en ejercicio, como los programas de formación inicial para futuros profesores, deben comprender en todos los elementos de capacitación, experiencias enriquecidas con TIC. Los estándares y recursos del proyecto "Estándares UNESCO de Competencias TIC para Docentes" (EDC-TIC) ofrecen orientaciones destinadas a todos los docentes y más concretamente, directrices para planear programas de formación del profesorado y selección de cursos que permitirán prepararlos para desempeñar un papel esencial en la capacitación tecnológica de los estudiantes.

En lo referente al argumento fundamental para seguir manteniendo las expectativas en el potencial educativo de las TIC, pese a lo limitado de los efectos documentales hasta el momento, es por las ventajas que ofrece como herramienta para pensar, sentir y actuar. Este argumento se apoya en la naturaleza simbólica de las tecnologías de información y comunicación en general, y de las tecnologías digitales en particular, es decir, en las posibilidades inéditas que ofrecen para acceder a la información, representarla, procesarla, transmitirla y compartirla.

En realidad, la novedad de las "nuevas" TIC o TIC digitales no reside en su naturaleza de tecnología para la información y la comunicación. Los seres humanos han utilizado siempre diversas tecnologías para transmitir información, comunicarnos y expresar ideas, sentimientos, emociones y deseos, desde las señales o símbolos tallados en la piedra o en la corteza de los árboles y las señales de humo, hasta el telégrafo, el teléfono, la radio o la televisión, pasando por los gestos y los movimientos corporales, el lenguaje de signos, el lenguaje oral, la lengua escrita o la imprenta.

Entonces la novedad tampoco reside en la introducción de un nuevo sistema simbólico para manejar la información, ya que los recursos semióticos que se encuentran en las pantallas de los ordenadores son básicamente los mismos que en un aula convencional: letras y textos escritos, imágenes fijas o en movimiento, lenguaje oral, sonidos, datos numéricos, graficas, etc. La novedad, en definitiva, reside más bien en el hecho de que las TIC digitales permiten crear entornos que integran capacidades humanas para representar, procesar, transmitir y compartir grandes cantidades de información sin limitaciones de espacio y tiempo, de forma casi instantánea y con un mínimo costo económico.

Ahora bien, todas las TIC, digitales o no, sólo proporcionan instrumentos psicológicos cuando su potencialidad semiótica es utilizada para planificar y regular la actividad y los procesos psicológicos propios y ajenos. En este sentido, la potencialidad semiótica de las TIC digitales es sin duda enorme. $Y$, en consecuencia, su potencialidad como 
instrumentos mediadores de los procesos intra e inter mentales implicados en la enseñanza y aprendizaje también lo es.

Efectivamente, los usos que los participantes hagan de las TIC dependerán, en buena medida, de la naturaleza y características del equipamiento y de los recursos tecnológicos puestos a su disposición. En este primer nivel, en el diseño tecnológico, lo que cuenta son las posibilidades $y$ limitaciones que ofrecen esos recursos para representar, procesar, transmitir y compartir información.

En cuanto a las aplicaciones de software informático y telemático, herramientas de navegación, de representación del conocimiento, de construcción de redes semánticas, hipermedia, bases de datos, sistemas expertos, de elaboración de modelos, de visualización, de comunicación sincrónica y asincrónica, de colaboración y elaboración conjunta, micro mundos, etc. varían enormemente según las posibilidades y limitaciones de los recursos disponibles. $Y$ estas variaciones, a su vez, establecen restricciones distintas en cuanto a las actividades y tareas que mediante el uso de dichas aplicaciones, van a poder desarrollar profesores y sus estudiantes para organizar las actividades de enseñanza y aprendizaje.

En vista de la aproximación que se está haciendo al estudio de un proceso formativo concreto que incorpora las TIC, su diseño tecnológico resulta prácticamente indisociable de su diseño pedagógico o instruccional, lo que conduce al segundo nivel de análisis propuesto. En efecto, los entornos de enseñanza y aprendizaje que incorporan las TIC, proporcionan una serie de herramientas tecnológicas, de recursos y de aplicaciones de software informático y telemático que sus usuarios potenciales pueden utilizar para aprender y enseñar.

Por lo general, las herramientas tecnológicas van acompañadas de una propuesta más o menos explícita, global y precisa según los casos, sobre la forma de utilizarlas para la puesta en marcha y el desarrollo de actividades pedagógicas. Lo que los usuarios suelen encontrar, son propuestas que integran tanto aspectos tecnológicos como instruccionales y que adoptan la forma de un diseño tecno instruccional con los siguientes elementos: una propuesta de contenidos, objetivos y actividades de enseñanza y aprendizaje, así como orientaciones y sugerencias sobre la manera de llevarlas a cabo.

Conviene subrayar que la incorporación de herramientas tecnológicas a la planificación de un proceso formativo, incluye siempre una serie de normas y procedimientos de uso, más o menos explícitos y formalizados de las herramientas incorporadas. Estas normas y procedimientos de uso son elementos esenciales del diseño tecno pedagógico y constituyen el referente inmediato a partir del cual los participantes utilizan las herramientas tecnológicas con el fin de organizar su actividad conjunta en torno a los contenidos y tareas de enseñanza y aprendizaje.

Respectivamente, la propuesta que aquí se muestra para el ámbito de las TIC está enmarcada en la teoría de J. Sarramona (2004) tomada de Guarapano y Martínez (2012). La propuesta está estructurada en cinco campos de acción: 
- Los sistemas informáticos (hardware, redes, software).

- El sistema operativo.

- Uso de internet.

- Uso de programas básicos.

- Actitudes necesarias con las TIC.

Se observa que, los dos primeros campos pretenden facilitar los conocimientos generales indispensables para enfrentarse exitosamente con la manipulación de las TIC. El tercero está centrado en el uso de internet, el medio de información y comunicación que ya resulta indispensable en la sociedad actual. El cuarto campo se enfoca en el uso de aquellos programas que es preciso conocer para actuar con soltura ante un ordenador, es decir, el procesador de textos, el editor gráfico, la hoja de cálculo y la base de datos. Finalmente, se toman en consideración las consecuencias morales del uso de las TIC puesto que se trata de fomentar su uso desde una perspectiva educativa y no meramente funcional.

El siguiente punto a tratar en este discurso está orientado a describir los fundamentos legales que soportan al sistema Educativo. Los mismos se sustentan en primer lugar, en la Constitución de la República Bolivariano Venezuela (1999), máximo instrumento legal y rector del desarrollo y la convivencia en el país, donde se consagran y profundizan los principios que consideran a la educación y la cultura como derechos fundamentales de todos los venezolanos. La Ley de Universidades (1970) y el Fundamento Legal de las Políticas Académicas Curriculares de la Universidad de Carabobo (2011). En este marco legal se toman los siguientes artículos considerando los criterios constitucionales más relevantes en materia educativa:

La Constitución de la República Bolivariana de Venezuela (1999), Artículo 9:

El estado reconocerá la autonomía universitaria como principio y jerarquía que permite a los profesores(as), estudiantes(es), egresados (as) de su comunidad dedicarse a la búsqueda del conocimiento a través de la investigación científica humanística, tecnológica, para beneficio espiritual y material de la nación. Las universidades autónomas se darán de normas de gobierno, funcionamiento y la administración eficiente de su patrimonio bajo el control y vigilancia que a tales efectos establezca la ley. Se consagrará la autonomía universitaria para planificar, organizar, elaborar y actualizar los programas de investigación, docencia y extensión.

\section{Artículo 37:}

Es función indeclinable del Estado la formulación, regulación, seguimiento y control de gestión de las políticas de formación docente a través del órgano con competencia en materia de Educación Universitaria, en atención al perfil requerido por los niveles y modalidades del Sistema Educativo y en correspondencia con las políticas, planes, programas y proyectos educativos emanados del órgano con competencia en materia de educación básica, en el marco del 
desarrollo humano, endógeno y soberano del país. La formación de los y las docentes del Sistema Educativo se regirá por la ley especial que al efecto se dicte y deberá contemplar la creación de una instancia que coordine con las instituciones de educación universitaria lo relativo a sus programas de formación docente.

\section{Artículo 102:}

La educación es un derecho humano y un deber social fundamental, es democrática, gratuita y obligatoria. El Estado al asumirla como función indeclinable y de máximo interés en todos sus niveles $y$ modalidades y como instrumento del conocimiento científico, humanístico y tecnológico al servicio de la sociedad. La educación es un servicio público y está fundamentada en el respeto a todas las corrientes del pensamiento con la finalidad de desarrollar el potencial creativo de cada ser humano y el pleno ejercicio de su personalidad en una sociedad democrática basada en la valoración ética del trabajo $y$ en la participación activa consciente y solidaria en los procesos de transformación social, circunstanciados con los valores de la identidad nacional y con una visión latinoamericana y universal.

La ley de Universidades (1970), Artículo 3:

Las Universidades deben realizar una función rectorar en la educación, la cultura y la ciencia. Para cumplir esta misio, sus actividades se dirigirán a crear, asimilar y difundir el saber mediante la investigación y la enseñanza; a completar la formación integral iniciada en los ciclos educacionales anteriores; y a formar los equipos profesionales y técnicos que necesita la Nación para su desarrollo y progreso.

Una vez descritos los fundamentos teóricos, antecedentes y marco legal que ampara al sistema educativo, y una vez conocido el contexto en el que se desarrolló esta investigación, es preciso exponer a continuación las estrategias metodológicas que permitieron planificar y organizar las acciones para alcanzar los objetivos propuestos.

\section{MÉTODO}

Ya formulado el problema de investigación, delimitado sus objetivos $\mathrm{y}$ asumidas las bases teóricas que orientarán el sentido de la misma, se seleccionaron los distintos métodos y técnicas que permitieron la obtención de los datos. En esta parte se incluyen las fases en que se desenvolvió el estudio y las técnicas de análisis de datos, es decir se plantea el marco metodológico que permite proyectar los instrumentos que serán necesarios para desarrollar la investigación.

Tomando en cuenta las características generales del estudio y los objetivos propuesto del mismo, para el presente trabajo se contempla el tipo de investigación descriptiva, la cual según Palella y Martins. (2010): "se ocupa de 
recolectar datos, describir y analizar su incidencia e interacción en un momento dado, sin manipularlas" (p. 104).

A su vez, la presente investigación presenta un diseño de campo, no experimental que según lo indica Palella y Martins (2010) "se realiza sin alterar las variables. Se observan los hechos tal y como se presentan en su contexto real en un tiempo determinado o no, para luego analizarlos" (p. 96).

Asimismo, la población según Balestrini (2006) "es el conjunto de elementos que se desean conocer algunas de sus características" (p. 40). En el caso de la presente investigación, la población objeto de estudio, estuvo representada por catorce (14) estudiantes, cursantes de las asignaturas: Seminario de Proyecto de investigación y Trabajo Especial de Grado de la Mención de Física de la Facultad de Ciencias de la Educación de la Universidad de Carabobo. Para la asignatura Seminario de Proyecto de Investigación la población constó de seis (6) estudiantes que comprenden la sección única de la tarde de los cuales efectivamente se cuenta con 4 debido a que dos miembros del grupo desertaron. Mientras que en la asignatura Trabajo Especial de Grado la población constó de ocho 8 estudiantes de la sección única de la tarde de los cuales se cuenta con 6 debido a que un integrante de grupo es el investigador y otro integrante desertó en la asignatura. Teniéndose una población total de diez estudiantes por ambas asignaturas.

En cuanto a la muestra, Según Palella y Martins (2010), representa un subconjunto de la población, accesible y limitado, sobre el que se realizan las mediciones o el experimento con la idea de obtener conclusiones generalizables a la población. En la presente investigación estuvo conformada por diez (10) estudiantes que representan el $100 \%$ del universo poblacional.

Durante el desarrollo de la presente investigación se llevaron a cabo varias etapas previamente planificadas, con la finalidad de presentar los resultados confiables y que pudieran servir de antecedentes valederos. A continuación, se mencionan las distintas fases en que se puede dividir el trabajo realizado, una vez que se planteó el problema y los objetivos:

- Selección de los sujetos.

- Selección del instrumento ya validado en investigación realizada por Guarapano y Martínez (2012) "Competencias del docente en formación en el uso de las tecnologías de información y comunicación caso: estudiantes de Práctica Profesional III de la mención de Matemática de la Facultad de Ciencias de la Educación de la Universidad de Carabobo.

- Aplicación de los instrumentos al grupo piloto

- Análisis de los resultados

- Conclusiones y recomendaciones

Continuando con la ruta metodológica, la técnica de recolección de datos según Palella y Martins (2010) "Se entenderá como, el procedimiento o forma particular de obtener datos o información" (P. 126). Es por ello que la técnica utilizada para esta investigación fue una encuesta que de acuerdo a Palella y Martins (2010), es una 
técnica destinada a obtener datos de Varias personas cuyas opiniones interesan al investigador. (p. 134)

Para la obtención de datos en este estudio, se usó como instrumento el cuestionario realizado por Guarapano y Martínez (2012) en la cual se empleó una escala de Likert, que según Palella y Martins (2010), consiste en un conjunto de ítems presentados en forma de afirmaciones o juicios ante los cuales se pide la reacción de los sujetos a quienes se administra. Dicho cuestionario consta de 35 ítems con cinco alternativas de respuestas: nunca, casi nunca, algunas veces, casi siempre $y$ siempre. La finalidad de dicha escala fue la de conocer las competencias relacionadas con técnicas de información y comunicación (TIC) que tienen los docentes en formación de las asignaturas Seminario Proyecto de Investigación y Trabajo Especial de Grado de la Mención de Física en la Facultad de Ciencias de la Educación de la Universidad de Carabobo.

Cabe destacar que todo instrumento debe cumplir con el requisito de la validez. Landeau (2010) la define como "el grado en el que el instrumento proporciona datos que reflejen realmente los aspectos que interesan estudiar". (p. 81) Mientras que, la confiabilidad "es el grado con el cual el instrumento prueba su consistencia, por los resultados que produce al aplicarlo repetidamente al objeto de estudio". Se puede decir entonces, que la confiabilidad es la capacidad que tiene el instrumento de registrar los mismos resultados y dar fe de que los datos son confiables.

Atendiendo a que el instrumento se encontraba validado previamente y se había aplicado ya la confiabilidad, es necesario reconocer en este estudio que los autores Guarapano y Martínez (2012) obtuvieron una confiabilidad de 0.887 dentro de la escala Alfa de Cronbach, porque se puede asegurar que es confiable y no amerita de ninguna modificación. Para la presente investigación, los estudios de confiabilidad no se realizaron debido a que el tamaño de la muestra es el total de la población de estudio.

Aunado a esto, los resultados obtenidos en este estudio se analizaron utilizando la Estadística Descriptiva, que según Palella y Martins (2010), "Consiste en la presentación de datos en forma de tablas y gráficos, la cual está diseñada para resumirlos o describirlos sin factores pertinentes adicionales; esto es, sin intentar inferir nada que vaya más allá de los datos vistos como tales (p.189).

Referente al análisis de datos obtenidos a través del cuestionario aplicado, se tabularon los ítems correspondientes, apoyado en el programa SPSS versión 18.0 bajo ambiente Linux, desarrollando las dimensiones que en este caso son los estratos de las materias pertenecientes a la investigación. De igual forma la representación del análisis de los datos obtenidos para reflejar las competencias, se fundamentó en el uso de tablas y de gráficas de columna, facilitando así su comprensión y mejor entendimiento en la aplicación del instrumento. Se elaboró un texto narrativo por cada dimensión expresada en tablas y gráficas, así como también de cada competencia y con el apoyo se dio respuesta a los objetivos planteados en la investigación. Finalmente 
se redactaron los resultados que se exponen en el siguiente segmento.

\section{RESULTADOS Y DISCUSIÓN}

Una vez realizado el estudio acerca de las competencias Tecnológicas de Información y Comunicación (TIC) de los docentes en formación de la mención de física de la FACE- UC. Caso: Estudiantes de Seminario Proyecto de Investigación y Trabajo Especial de Grado de la mención de física en la facultad de ciencias de la educación de la Universidad de Carabobo, se procedió a realizar un análisis por dimensión, de estos se pudo concluir que:

En la dimensión "los sistemas informáticos" la mayoría de los encuestado manifiestan poseer esta competencia, esto se evidencia debido a que de los estudiantes encuestados un $62,5 \%$ se encuentran por encima del promedio. Por otra parte, el ítem 3 referente a utilizar adecuadamente la terminología relacionada con los sistemas operativos presentó una alta tendencia negativa, así como también el ítem 4 presentó una tendencia positiva.

Con respecto al estudio de datos de la dimensión "uso del internet" los estudiantes manifestaron poseer esta competencia ya que $75 \%$ de los encuestados se encuentran por encima del promedio. Por otra parte, el ítem 8 , referente a realizar búsquedas avanzadas en internet utilizando filtros de múltiples palabras claves y algún operador lógico, alcanzó un promedio perfecto de 5 .

Concerniente a la dimensión "Uso de Programas básicos" se observó que 62,5\% de los encuestados se encuentran por encima del promedio demostrando que manifiestan poseer esta dimensión. Se destacó que en el ítem 13 donde se detecta si el encuestado reconoce la terminología de los editores de texto (tablas, numeración y viñetas, notas, etc.) así como también en el ítem 15, que reconoce las limitaciones de las herramientas de corrección ortográficas, representan un porcentaje muy alto de respuestas con tendencia negativa, esto expresa la necesidad de fomentar jornadas de alfabetización digital en cuanto al uso de programas básicos.

Referente al análisis de los datos de la dimensión "actitud necesaria con las TIC" los estudiantes encuestados manifestaron poseer esta competencia. La misma se evidencia en el hecho de que $79,16 \%$ de los estudiantes se encuentran por encima del promedio. En total, los ítems con mayor tendencia positiva fueron el 25 y 30 referentes a mantener un cierto control del tiempo de utilización de los juegos y otros entretenimientos que proporcionan las TIC y reconocer los riesgos y consecuencias de descargar software ilegal. Por otra parte, el ítem con mayor tendencia negativa fue el referido a valorar críticamente las posibilidades e inconvenientes que ofrece las nuevas tecnologías y las repercusiones que tienen en la vida cotidiana.

\section{CONCLUSIONES}

Con respecto a la dimensión "los sistemas informáticos" los estudiantes manifestaron no poseer dicha competencia debido a que $58,4 \%$ de los encuestados se encuentran por debajo del promedio. Esta situación pone de manifiesto la necesidad de promover jornadas de alfabetización digital 
en la mención de física de la facultad de educación e incentivar al uso de la tecnología en los procesos de enseñanza y aprendizaje.

En el caso de la dimensión "uso del internet" se observó que un $73,3 \%$ de los encuestados manifestaron poseer esta competencia. En el ítem 10 relacionado con el uso de manera responsable las diversas herramientas telemáticas de intercambio y comunicación grupal, se alcanzó un promedio de 5 , lo que significa que los encuestados presentan una alta tendencia positiva.

En lo que respecta a la dimensión "uso de programas básicos" los estudiantes manifestaron poseer esta competencia lo cual se evidencia en que $73,6 \%$ de los encuestados que se encuentran por encima del promedio. Para esta dimensión los ítems 14, 17, 18,19, referentes a: realizar una búsqueda dentro de un documento; modificar los atributos de una imagen; reconoce la terminología básica sobre hojas de cálculo; reconoce los datos y las fórmulas de las celdas, distinguiendo los datos introducidos de los calculados, etc. presentaron una alta tendencia positiva lo cual se puede definir como una fortaleza.

Referente al análisis de los datos para la dimensión "actitud frente a las TIC" se detectó que el $76,4 \%$ de los estudiantes que manifestaron poseer esta competencia presentaron un promedio por 4.03 por encima de la media. Para esta dimensión el ítem 31, referente a: actuar con prudencia en el uso de las TIC: procedencia de los mensajes del correo, archivos críticos, etc. se destaca por presentar un 83,3\% por encima del promedio.

En suma, se puede afirmar que los docentes en formación de la mención de física de la Universidad de Carabobo poseen las competencias básicas tecnológicas, sin embargo, se requiere de un reforzamiento en cuanto a todas las dimensiones mencionadas. A partir de este estudio se puede realizar investigaciones posteriores con el fin de promover cursos de capacitación perfeccionamiento en manejo de las TIC en el campo educativo ya que las mismas representan el nuevo panorama de las metodologías de enseñanza aprendizaje.

\section{REFERENCIAS}

Arias, F. (2012). El proyecto de investigación, introducción a la metodología científica. (6ta Ed). Caracas, Venezuela: Episteme

Balestrini, M. (2006). Como elaborar un proyecto de investigación. Caracas: Consultores Asociados

Cabero, J. y Lloverante, M. (2005). Las plataformas virtuales en el ámbito de la teleformación. Recuperado de http://www.ealternativas

Coll. C. (2001). Psicología de la educación virtual. (1era Ed.). Madrid: Morata.

Constitución de la República Bolivariana de Venezuela. (1999). Gaceta Oficial de la República Bolivariana de Venezuela, 5.453, marzo 3, 2000

Corredor, C. P. G., \& Rincón, J. C. V. (2014). Movimientos sociales e internet. Editorial Pontificia Universidad Javeriana.

Galvis, A. (1992). Ingeniería de software educativo. [libro en línea]. Disponible en: es.slideshare.net/adrianaaraquerangel/ga Ivis-por-joe-30604090. [Consulta 2014, Julio 15]

Guarapano y Martínez. (2012). Competencias del docente en formación en el uso de las tecnologías de información y comunicación caso: estudiantes de Práctica Profesional III de la mención de Matemática de la Facultad de Ciencias de la Educación de la Universidad de Carabobo 
Ordoñez, C.; Góngora, I.; Ordoñez, B.; Rico, C. y Cerón P. (2010). Implementación de la tecnología computacional en el aprendizaje de la matemática y la física. Disponible;

http://www.slideshare.net/pabloedilsonc /implementacion-de-tic-en-la-enseanzade-la-fisica-y-la-matematica. [Consultada: 2013, 10 febrero]
Palella, S. y Martins, F. (2010). Metodología de la investigación cuantitativa. Venezuela: Editorial FEDUPEL

Unesco (2008). Estándares UNESCO de competencia en TIC para docentes Recuperado de: http://eduteka.icesi.edu.co/articulos/Esta ndaresDocentesUnesco [Consulta julio 2014] 